\title{
Breast Cancer Among Patients Below Age 40: Clinicopathological Features and Survival Results
}

\author{
Berrin Benli YAVUZ, Meryem AKTAN, Gul KANYILMAZ \\ Necmettin Erbakan University, Meram Faculty of Medicine, Department of Radiation Oncology, Konya, TURKEY
}

\begin{abstract}
Among young women breast cancer exhibits quite a heterogenous, quite agressive and complex biology. The purpose of this study is to describe clinicopathological features that affect survival ratio among breast cancer women below age- 40 . 803 patients having received adjuvant radiotherapy were retrospectively analyzed. Patients were categorized under two groups: age $\leq 40$ and age $>40$. Treatments and clinicopathological features were analyzed. 19.4\% (156) of 803 patients were below age 40. In patient group below age 40, more neoadjuvant chemotherapy (NAC) was administered compared to the patient group above age 40 ( $p=0.007$ ) and it was detected that there were higher incidences of stage 3 disease $(p=0.011)$, more advanced nodal disease (N2-3) $(p=0.046)$ and more metastasis $(p<0.001)$. In conducted multivariate analysis for all age groups presence of N2-3 disease ( $p=0.011)$ and in below age-40 group being grad $3(p=0.025)$ was found to affect overall survival (OS) negatively. In disease free survival (DFS)-focused analysis; for all age groups, receiving NAC ( $p=0.001)$, presence of $N 2-3$ disease $(p=0.002)$ and being below age 40 were found to have a negative effect; in below age-40 group presence of NAC $(p=0.013)$ and perineural invasion $(p=0.035)$; in above age-40 group receiving NAC $(p=0.023)$ and presence of N2-3 disease $(p=0.035)$ had a negative effect. Being below age 40 is an independent prognostic factor for DFS. It is suggested to conduct further studies on specific tumor biology to analyze the same group with respect to the characteristics of more aggressive tumors.
\end{abstract}

Keywords: Breast cancer, Young age, Prognosis, Survival

\section{INTRODUCTION}

Breast cancer is a frequent malignancy witnessed among women ${ }^{1}$ and its frequency is expected to climb further in the next 5-10 years. ${ }^{2}$ According to SEER database, between 2013-2017 years breast cancer was diagnosed among 446.594 female cases. Among women below age 40, incidence of breast cancer is 105.6/100000 and compared to female patients above age 40 this is a significantly low ratio. ${ }^{3}$ Yet, breast cancer is the most common cause of cancer-induced deaths among women below age $40 .^{2,4,5}$

For breast cancer patients, young age is defined as an independent indicator for bad prognosis. ${ }^{6}$ There is yet not a conclusive definition of young age in literature. As the cut off value, ages 35, 40, 45 are widely chosen. In our study we chose this value as age 40 .
It has been reported that young age is related with more advanced stage, more aggressive tumor characteristics, higher mortality and recurrent ratios. ${ }^{7,8}$ Since in this period breast tissue is denser and there is the possibility of mistaking with lactation and pregnancy stages and absence of screening in the routine schedule of this age group, diagnosis can only be given in advanced stages. ${ }^{7,9}$

In a population-based study by Kroman et al. among 10.356 patients, multivariate analysis were conducted to reveal independent effect of age and age 45-49 was accepted as reference range. It was demonstrated that in the group below age 35, death risk increased by 1.46 times and in the group between the age range of 35-39, the same risk multiplied by 1.26 times. $^{9}$ 
In this study conducted in one center only, the aim is to draw a retrospective analysis between young women ( $\leq 40$ age) and women above age 40 who were treated under the same protocol to detect if any differences existed in terms of tumor and treatment characteristics as well as prognosis.

\section{PATIENTS AND METHODS}

\section{Patient Selection}

803 patients who received adjuvant radiotherapy in our clinic between January 2010 - July 2019 were retrospectively analyzed. This study included female patients who were; above age 18 , non-metastatic, whose invasive carcinoma diagnosis was histopathologically verified, who received curative treatment and were monitored for a period of minimum 1 year. Cases with another concurrent cancer, a incomplete lymph node dissection and bilateral breast carcinoma were excluded from the study. Patients were staged according to American Joint Committee on Cancer (AJCC) 8. version. ${ }^{10}$ In patients receiving neoadjuvant treatment, staging was made according to screening methods before treatment. In patients who received surgery first staging was based on pathology reports.

Patients were categorized under two groups: age $\leq$ 40 and age $>40$. Characteristics of the patient and the tumor were retrieved from relevant medical records. Treatments received by the patients (surgery type (breast conserving surgery/mastectomy), having chemotherapy (CT), radiotherapy (RT) fields and relevant doses), clinicopathological features (histological type, grade, stage, tumor size, lymph node involvement, lymphovascular invasion (LVI), perineural invasion (PNI), extracapsular extension (ECE), estrogen/progesterone receptor positivity (ER/PR) and HER-2 condition) were independently examined for both groups.

Prior to conducting the study, approval of Ethics Committee was taken and ethical principles in the last version in Declaration of Helsinki were likewise observed. Due to the nature of this study, informed consents of the patients were forsaken.

\section{Treatment}

Surgically patients were given breast conserving surgery (BCS) or modified radical mastectomy
(MRM), axillary dissection or sentinal lymph node sampling. According to the stages of patients, 4 cures of cyclophosphamide and adriamycin +/- 12 weeks or 4 cure of taxane-group chemotherapy was administered as adjuvant or neoadjuvant therapy.

After BCS adjuvant, RT was given to all cases. After MRM, adjuvant RT was given in the presence of T3-4 tumor, lymph node positivity, presence of ECE, presence of LVI or PNI. In patients who would receive regional lymph irradiation, supraclavicular region irradiation was included into the treatment. For inner quadrant tumors and patients having many involved lymph nodes, mamaria interna irradiation was included into the treatment protocol. Standard two parallel opposing tangential fields and 3 dimensional conformal RT techniques were applied for a total dose of $50 \mathrm{~Gy}$. In BCS-administered cases 10-16 Gy boost dose was added. In all patients, the Eclipse treatment planning system (Varian Medical Systems Inc., Palo Alto, CA) was followed in the treatment procedure.

\section{Survival}

Primary goal of this research is to analyze whether or not young age is an independent prognostic factor among breast cancer women. Follow-up period of the cases was computed as the period lasting from date of diagnosis till final control of the disease or patient's death. Overall survival (OS) related to the period between date of diagnosis till death; disease-free survival (DFS), on the other hand, was defined as the period until the realization of metastasis/recurrent/death distant from hand date of diagnosis.

Patients were followed up till death or March 2020. As a routine procedure, follow-ups were conducted every 3 months in the first 2 years, every 6 months till 5 years and annually in following years.

\section{Statistical Analysis}

For categorical variables, patient characteristics were summarized as $\mathrm{n}(\%)$ whereas for continuous variables they were summarized as median. Tumor and patient characteristics as well as treatment features between two subgroups were examined by utilizing Chi-square test. Survival ratios were measured by harnessing Kaplan-Meirer method. 


\begin{tabular}{|c|c|c|c|}
\hline Charecteristics & $\leq \mathbf{4 0}$ years $n(\%)$ & $>40$ years $n(\%)$ & $P$ value \\
\hline Patients & $156(19.4)$ & 647 (80.6) & \\
\hline Age (median) & $35.5(27-40)$ & $53(41-82)$ & \\
\hline \multicolumn{4}{|l|}{ Molecular subtype } \\
\hline Luminal A & $90(57.7)$ & $419(64.8)$ & 0.268 \\
\hline Luminal B & $33(21.2)$ & $98(15.1)$ & \\
\hline HER/neu enriched & $14(9)$ & $59(9.1)$ & \\
\hline Basal-like & $19(12.2)$ & $71(11)$ & \\
\hline \multicolumn{4}{|l|}{ Stage } \\
\hline Stage I-II & 85 (54.5) & $423(65.4)$ & $0.011^{*}$ \\
\hline Stage III & $71(45.5)$ & 224 (34.6) & \\
\hline \multicolumn{4}{|l|}{ Tumour stages } \\
\hline T0-1-2 & $122(78.2)$ & 541 (83.6) & 0.11 \\
\hline T3-4 & $34(21.8)$ & $106(16.4)$ & \\
\hline \multicolumn{4}{|l|}{ Lymph node stages } \\
\hline No-1 & 95 (60.9) & 448 (69.2) & $0.046^{*}$ \\
\hline N2-3 & $61(39.1)$ & $199(30.8)$ & \\
\hline \multicolumn{4}{|l|}{ Grade } \\
\hline Grade 1-2 & $113(80.1)$ & $432(73.7)$ & 0.114 \\
\hline Grade3 & 28 (19.9) & $154(26.3)$ & \\
\hline \multicolumn{4}{|l|}{ ECE } \\
\hline Yes & $53(40.5)$ & $196(35.8)$ & 0.32 \\
\hline No & $78(59.5)$ & $351(64.2)$ & \\
\hline \multicolumn{4}{|l|}{$\mathrm{PNI}$} \\
\hline Yes & 39 (32.2) & $176(30.6)$ & 0.72 \\
\hline No & $82(67.8)$ & $399(69.4)$ & \\
\hline \multicolumn{4}{|l|}{ LVI } \\
\hline Yes & $63(52.1)$ & $270(46.5)$ & 0.26 \\
\hline No & $58(47.9)$ & 311 (53.5) & \\
\hline \multicolumn{4}{|c|}{ Neoadjuvant chemotherapy } \\
\hline Yes & $18(11.5)$ & $36(5.6)$ & $0.007^{*}$ \\
\hline No & $138(88.5)$ & $611(94.4)$ & \\
\hline \multicolumn{4}{|l|}{ Chemotherapy } \\
\hline Yes & $149(95.5)$ & $587(90.7)$ & 0.052 \\
\hline No & $7(4.5)$ & $60(9.3)$ & \\
\hline \multicolumn{4}{|l|}{ Surgery type } \\
\hline MRM & $102(65.4)$ & $374(57.8)$ & 0.084 \\
\hline BCS & $54(34.6)$ & $273(42.2)$ & \\
\hline \multicolumn{4}{|l|}{ Metastasis } \\
\hline Yes & $46(29.5)$ & $72(11.1)$ & $<0.001^{*}$ \\
\hline No & $110(70.5)$ & 575 (88.9) & \\
\hline $\begin{array}{l}\text { ECE }=\text { extracapsular extens } \\
\text { BCS= breast conserving sL } \\
\text { * Statistically significant }\end{array}$ & ohovascular invasion; P & al invasion; MRM= moc & \\
\hline
\end{tabular}

Survival variances between two groups were analyzed by log-rank test path. Cox proportional hazard models were employed in univariate and multivariate analysis. In univariate analysis $p<0.20$ values were included in multivariate analysis. $\mathrm{P}<$
0.05 was taken as the statistically significant value. All of the statistical analyses were conducted by employing Version 13 of SPSS (Statistical Package for Social Sciences). 

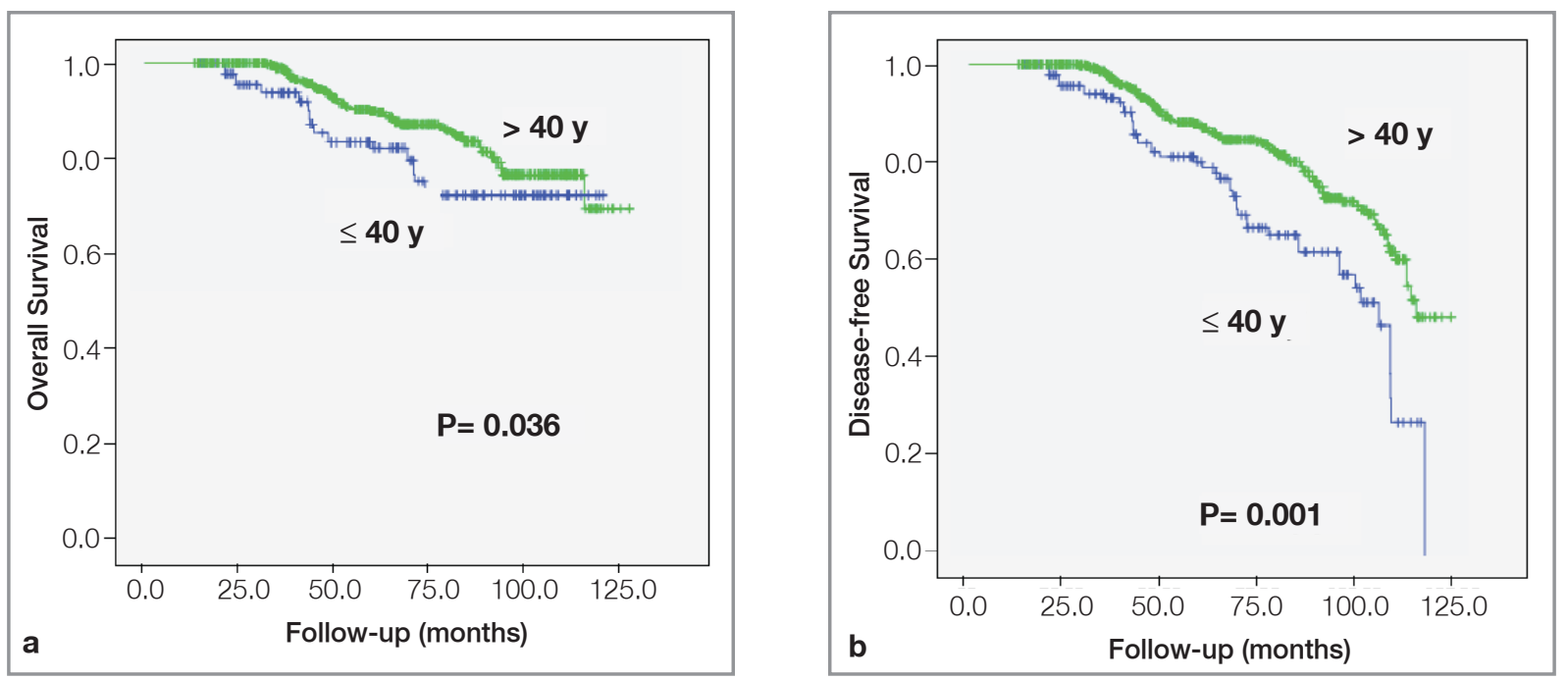

Figure 1. Overall survival (a) and disease free survival (b) by age groups: $\leq 40$ vs $>40$

\section{RESULTS}

\section{Characteristics of the Patients and Treatment}

$19.4 \%$ (156) of a total of 803 patients were below age 40. Median length of follow-up was 61.86 months (13.17-127.77). In below age-40 group median age was 35.5 (27-40), in above age-40 group it was 53 (41-82). Characteristics of the patients and treatment are as exhibited in Table 1. In below age-40 group, more neoadjuvant $\mathrm{CT}$ was applied than above age-40 patient group (11.5\% vs. $5.6 \% ; p=0.007)$. In young patient group stage 3 disease $(p=0.011), N 2-3$ disease $(p=0.046)$ and presence of metastasis $(p<0.001)$ were detected to be more frequent incidences. Between both groups grade, T stage, ECE presence, PNI presence, LVI presence, molecular subtype (luminal A, luminal B, Her/neu enriched, basal-like) which are common prognostic factors in breast cancers were not significantly different. In group below age 40, 149 patients $(95.5 \%)$ received CT. In other group 587 (90.7\%) patients received CT $(\mathrm{p}=0.052)$. Not any significant difference was detected in terms of surgery type, radiotherapy dose and field.

\section{Survival Analysis}

Mean survival was 103.1 months in below age-40 group and 114.08 months in above age-40 group. 2, 5,10 years of OS ratios were respectively ordered as $95.8 \%, 83.9 \%, 75 \%$ and $100 \%, 91 \%, 72.5 \%$ in below age 40 and above 40 group. 2, 5, 10 years of DFS ratios were respectively ordered as $95.9 \%$, $81.6 \%, 64.4 \%$ and $100 \%, 89 \%, 52 \%$. In all age groups, age effect on OS and DFS was analyzed. In the group below age-40 survival mean was 103.1 months while in $>40$ group it was 114.088 months $(\mathrm{p}=0.036)$ and disease-free survival was 93.48 months and 108.630 months $(\mathrm{p}<0.001)$ (Figure $1 a-b)$.

In univariate analysis, for below age-40 group receiving neoadjuvant $\mathrm{CT}(\mathrm{NAC})(\mathrm{p}<0.001)$, being in stage $3(\mathrm{p}<0.001)$, having grade 3 pathology $(\mathrm{p}=$ $0.044)$, being T3-4 stage ( $p<0.001)$, being N2-3 stage $(\mathrm{p}<0.001)$, presence of ECE $(\mathrm{p}=0.029)$; for above age-40 group being in stage $3(\mathrm{p}<0.001)$, being in N2-3 disease $(\mathrm{p}<0.001)$, presence of ECE $(p=0.003)$, presence of LVI $(p=0.003)$ were found to be negative factors for OS. In the univariate analysis for molecular subtypes, OS was better in the Luminal-B subgroup than the other groups in the all age group and under 40 years of age. In the group over 40 years of age, survival of Luminal-B subgroup was better than the triple negative group $(p=0.020)$. In multivariate analysis, for all age groups being N2-3 disease and in below age-40 group being in grade 3 were found to be negative factors for OS (Table 2, 3, 4).

In univariate analysis conducted with respect to DFS, for below age-40 group, receiving NAC ( $\mathrm{p}<$ $0.001)$, having stage 3 disease $(\mathrm{p}=0.001)$, presence of T3-4 $(\mathrm{p}=0.035), \mathrm{N} 2-3(\mathrm{p}<0.001)$, ECE $(\mathrm{p}=$ 
International Journal of Hematology and Oncology

Table 2. Multivariate analysis of disease free survival and overall survival in patients $\leq 40$ years.

\begin{tabular}{|c|c|c|c|c|c|c|}
\hline & \multicolumn{3}{|c|}{ Overall Survival } & \multicolumn{3}{|c|}{ Disease Free Survival } \\
\hline & HR & $95 \% \mathrm{Cl}$ & $P$ value & HR & $95 \% \mathrm{Cl}$ & $P$ value \\
\hline Neoadjuvant CT (no/yes) & - & $0.00-$ & 0.993 & 5.668 & $1.438-22.329$ & $0.013^{*}$ \\
\hline ECE (no/yes) & 2.098 & $0.141-31.192$ & 0.591 & 1.741 & $0.407-7.443$ & 0.455 \\
\hline PNI(no/yes) & 1.785 & $0.342-9.326$ & 0.492 & 2.692 & $1.071-6.762$ & $0.035^{\star}$ \\
\hline Tumour stage (T0-1-2 vs T3-4) & 1.284 & $0.350-4.714$ & 0.706 & 2.028 & $0.620-6.635$ & 0.242 \\
\hline Nodal stage (N0-1 vs N2-3) & 3.657 & $0.327-40.970$ & 0.196 & 3.643 & $0.969-13.694$ & 0.056 \\
\hline Grade(grad1-2 vs grad 3) & 4.119 & $1.197-14.172$ & $0.025^{\star}$ & - & - & - \\
\hline Molecular subtype & & & & - & - & - \\
\hline Luminal A & 1 & & 0.540 & & & \\
\hline Luminal B & 0.255 & $0.030-2.137$ & 0.208 & & & \\
\hline Her/neu enriched & 0.000 & $0.00-7.831$ & 0.963 & & & \\
\hline Basal-like & 1.575 & $0.308-8.051$ & 0.585 & & & \\
\hline
\end{tabular}

$0.023)$; for above age-40 group receiving NAC ( $\mathrm{p}<$ $0.001)$, having stage 3 disease ( $\mathrm{p}<0.001)$, and presence of $\mathrm{N} 2-3(\mathrm{p}<0.001), \mathrm{ECE}(\mathrm{p}=0.015)$ and LVI $(p=0.007)$ were statistically significant. For DFS, no statistically significant difference was found between molecular subgroups in all age groups. In multivariate analysis for all age groups, receiving NAC, presence of N2-3 disease and age below 40; in below age- 40 group presence of NAC and PNI; in above age-40 group receiving NAC and having N2-3 disease were found to have negative effects (Table 2, 3, 4).

\section{Discussion and Conclusion}

According to GLOBACAN data, breast cancer is detected in one out of every four women over the world. ${ }^{11}$ Despite its rarity among women below age

\begin{tabular}{|c|c|c|c|c|c|c|}
\hline & \multicolumn{3}{|c|}{ Overall Survival } & \multicolumn{3}{|c|}{ Disease Free Survival } \\
\hline & HR & $95 \% \mathrm{Cl}$ & $P$ value & HR & $95 \% \mathrm{Cl}$ & $P$ value \\
\hline Neoadjuvant CT(no/yes) & 1.398 & $0.326-5.987$ & 0.652 & 2.646 & $1.146-6.104$ & $0.023^{*}$ \\
\hline ECE(no/yes) & 1.303 & $0.648-2.621$ & 0.458 & 1.143 & $0.655-1.996$ & 0.637 \\
\hline LVI(no/yes) & 1.904 & $0.947-3.830$ & 0.071 & 1.653 & $0.967-2.826$ & 0.066 \\
\hline PNI(no/yes) & 1.186 & $0.636-2.209$ & 0.592 & - & - & - \\
\hline Tumour stage (T0-1-2 vs T3-4) & 1.334 & $0.675-2.636$ & 0.407 & 1.206 & $0.675-2.154$ & 0.527 \\
\hline Nodal stage (N0-1 vs N2-3) & 2.074 & $1.023-4.205$ & 0.052 & 1.830 & $1.042-3.213$ & $0.035^{*}$ \\
\hline Molecular subtype & & & & - & - & - \\
\hline Luminal A & 1 & & 0.435 & & & \\
\hline Luminal B & 0.614 & $0.237-1.591$ & 0.315 & & & \\
\hline Her/neu enriched & 0.629 & $0.237-1.667$ & 0.351 & & & \\
\hline Basal-like & 1.312 & $0.609-2.826$ & 0.487 & & & \\
\hline
\end{tabular}


Table 4. Multivariate analysis of disease free survival and overall survival in all population

\begin{tabular}{|c|c|c|c|c|c|c|}
\hline & \multicolumn{3}{|c|}{ Overall Survival } & \multicolumn{3}{|c|}{ Disease Free Survival } \\
\hline & HR & $95 \% \mathrm{Cl}$ & $P$ value & HR & $95 \% \mathrm{Cl}$ & $P$ value \\
\hline Neoadjuvant CT (no/yes) & 1.546 & $0.532-4.495$ & 0.423 & 3.315 & $1.616-6.803$ & $0.001^{*}$ \\
\hline ECE (no/yes) & 1.244 & $0.645-2.398$ & 0.515 & 1.004 & $0.595-1.692$ & 0.989 \\
\hline LVI (no/yes) & 1.549 & $0.828-2.900$ & 0.171 & 1.212 & $0.739-1.988$ & 0.446 \\
\hline PNI (no/yes) & 1.067 & $0.621-1.834$ & 0.815 & 1.130 & $0.720-1.772$ & 0.595 \\
\hline Tumour stage (T0-1-2 vs T3-4) & 1.296 & $0.712-2.359$ & 0.396 & 1.017 & $0.601-1.720$ & 0.950 \\
\hline Nodal stage (N0-1 vs N2-3) & 2.329 & $1.213-4.473$ & $0.011^{*}$ & 2.332 & $1.370-3.968$ & $0.002^{*}$ \\
\hline Age $(\leq 40$ vs $>40)$ & 1.727 & $0.887-3.362$ & 0.108 & 1.673 & $1.041-2.686$ & $0.033^{*}$ \\
\hline Molecular subtype & & & & - & - & - \\
\hline Luminal $\mathrm{A}$ & 1 & & 0.112 & & & \\
\hline Luminal B & 0.503 & $0.213-1.190$ & 0.118 & & & \\
\hline Her/neu enriched & 0.524 & $0.202-1.356$ & 0.183 & & & \\
\hline Basal-like & 1.442 & $0.728-2.858$ & 0.294 & & & \\
\hline
\end{tabular}

40 , it still accounts for the biggest cause of cancerinduced deaths. ${ }^{2,45}$ Among young women breast cancer is associated with more advanced stage disease, more aggressive tumor characteristics, worse survival and local disease control. ${ }^{7,12}$ Findings of this study demonstrated that when compared with patients above age 40, mortality and disease-free survival ratios tend to be worse in women below age 40 . In below and above age 40 groups OS ratios were respectively computed as $17.3 \%$ and $11 \%(\mathrm{p}=0.041)$; disease-free survival ratios were ordered as $30.1 \%$ and $16.1 \%(\mathrm{p}<0.001)$. Among young women, more stage 3 disease, more advanced nodal stage (N2-3) and more metastasis were determined. A significant portion of patients in this age group applied to the physician at the start due to complaints of palpable mass or axillary lymph node. ${ }^{13}$ In below age-40 group, since there is lack of routine screening programs and protector measurements it is more likely to receive a diagnosis at more advanced stage. ${ }^{8,9}$ Some of the other potential causes for delayed diagnosis could be denser breast tissue and overlapping of this period with lactation and gestation periods. ${ }^{7,9}$

Although in most of the young age studies there is bad prognostic ${ }^{14,15,16}$, this effect could not be demonstrated in other studies. ${ }^{17}$ In a populationbased cohort study that compared women below and above age 40 it was displayed that $6.4 \%$ of a total of 243.012 breast cancer patients were below age 40 and in this same group larger and higher invasive tumors, more lymph node positivity and more local recurrent were demonstrated. ${ }^{7}$ Likewise in POSH study it was suggested that among young patients ER (-) tumor frequency, higher grade and risk for early disease recurrent were all higher. In our cohort study, 5 years of OS was $83.9 \%$ and similarly in this study the ratio was $82 \% .{ }^{18}$ However in a Singapore-based study, higher relative survival ratios were detected among women below age $35 .{ }^{19}$ This result may be since patient population of that study involved 6895 breast cancer women between 1968-1992 and probably administering a less effective treatment to the old age women.

Among women below age $3515-30 \%$ ratio of BRCA1-2 mutation can be detected. ${ }^{20}$ There are certain studies exhibiting the effect of BRCA condition on survival rates. In our study we could not analyze our patients' BRCA mutation and family disposition since we did not know personal data of all the patients and the study was a retrospective analysis. However the research by National Breast Cancer Registry Program (NBCRP) evidenced that among $33.9 \%$ of 19.503 female breast cancer cases there was a cancer story in family and among these cases $15.8 \%$ had a family story of breast cancer. In 
this study the ratio of patients below age 40 was $16.6 \% .{ }^{21}$ In the study conducted by Han et al., there was not a detectable difference in terms of family story between below and above age 35 groups. ${ }^{20}$

NAC inflammatory breast cancer is the standard treatment procedure for inoperable patients or patients with large tumors. ${ }^{22}$ In this age it can also be administered for early stage patients too. ${ }^{20} \mathrm{Re}$ gardless of its advantages, not a difference could be demonstrated for overall survival and recurrent trace survival rates in the conducted studies. ${ }^{23,24}$ Despite not being the major focus of our study, receiving NAC was found to be a crucial factor for disease-free survival in both univariate and multivariate analysis for all age groups. In the same vein, age below 40 was a negative factor for the overall survival as shown by univariate analysis. In this study, $81.5 \%$ of NAC-administered patients had stage 3 disease while in the group not receiving NAC it was measured as $33.5 \%(\mathrm{p}<0.001)$. Since in our hospital early stage disease is mostly treated under surgery at first, patients who received NAC had more advanced stage disease. Decrease in OS and DFS is considered to be linked with this detection.

Weak aspects of our study were its retrospective nature, partially-limited sampling quantity and unknown BRCA conditions. However, strong aspects were treating the patient group in the same center under the same treatment protocol and a follow-up length of median 61 months.

In conclusion; being below age 40 is an independent prognostic factor in disease-free survival. It has been detected that when compared with patients above age 40 , mortality and disease-free survival ratios tend to be worse in women below age 40 . In young patient group more advanced stage, more advanced nodal stage and presence of more metastasis were designated. It is thus suggested to conduct further studies on specific tumor biology to analyze the same group with respect to the characteristics of more aggressive tumors.

\section{REFERENCES}

1. Mustillo A, Ayoub JP, Charpentier D, et al. Prognosis in young women less than 40 years of age with brain metastasis from breast cancer. Curr Oncol 27: 39-45, 2020.
2. Anastasiadi Z, Lianos GD, Ignatiadou E, et al. Breast cancer in young women: an overview. Updates Surg 69: 313-317, 2020.

3. SEER DATA: Howlader N, Noone AM, Krapcho M, et al. SEER Cancer Statistics Review, 1975-2017, National Cancer Institute. Bethesda, MD, https://seer.cancer.gov/csr/1975 2017/, based on November 2019 SEER data submission, posted to SEER web site. Access date: April 2020.

4. Plichta J, Thomas SM, Vernon R, et al. Breast cancer tumor histopathology, stage at presentation, and treatment in the extremes of age. Breast Cancer Res Treat 180: 227-235, 2020.

5. Ribnikar D, Riberio JM, Pinto D, et al. Breast cancer under age 40: a Different approach. Curr Treat Options in Oncol 16: $16,2015$.

6. Limbach KE, Leon E, Pommier RF, Pommier SJ. Comparision of breast cancer incidence, clinicopathologic features, and risk factor prevalance in women aged 20-29 at diagnosis to those aged 30-39. Breast J 26: 1069-1070, 2020.

7. Gnerlich JL, Deshpande AD, Jeffe DB, et al. Elevated breast cancer mortality in young women ( $<40$ years) compared with older women is attributed to poorer survival in early stage disease. J Am Coll Surg 208: 341-347, 2009.

8. Radecka B, Litwiniuk M. Breast cancer in young women. Ginekol Pol 87: 659-663, 2016.

9. Kroman N, Jensen MB, Wohlfahrt J, et al. Factors influencing the effect of age on prognosis in breast cancer: population based study. BMJ 320: 474-478, 2000.

10. Amin MB, Edge S, Greene F, et al (eds). AJCC Cancer Staging Manual. 8th edition. Springer, 2017: 589-628.

11. Bray F, Ferlay J, Soerjomataram I, et al. Global cancer statistics 2018: GLOBACAN estimates of incidence and mortality worldwide for 36 cancers in 185 countries. CA Cancer J Clin 68: 694-424, 2018.

12. Wong FY, Tham WY, Nei WL, et al. Age exerts a continuous effect in the outcomes of Asian breast cancer patients treated with breast-conserving therapy. Cancer Common 38: 39, 2018.

13. Durhan $G$, Azizova $A$, Onder $O$, et al. Imaging findings and clinicopathological correlation of breast cancer in women under 40 years old. Eur J Breast Health 15: 147-152, 2019.

14. Love RR, Due NB, Dinh NV, et al. Young age as an adverse prognostic factor in premenopausal women with operabl breast cancer.Clin Breast Cancer 2: 294-298, 2002.

15. Peng R, Wang S, Shi $Y$, et al. Patients 35 years old or younger with operable breast cancer are more at risk for relapse and survival: A retrospective matched case-control study. Breast 20: 568-573, 2011.

16. Zhao Y, Dong X, Li R, Song J, Zhang D. Correlation between clinical-pathologic factors and long-term follow-up in young breast cancer patients. Transl Oncol 8: 265-272, 2015. 
International Journal of Hematology and Oncology

17. Rapiti E, Fioretta G, Verkooijen HM, et al. Survival of young and older breast cancer patients in Geneva from 1990 to 2001. Eur J Cancer 41: 1446-1452, 2005.

18. Copson E, Eccles B, Maishman T, et al. POSH Study Steering Group. Prospective observational study of breast cancer treatment outcomes for UK wpomen aged 18-40 years at diagnosis: The POSH study. J Natl Cancer Inst 105: 978-988, 2013.

19. Chia KS, Du WB, Sankaranarayanan R, et al. Do younger female breast cancer patients have a poorer prognosis? Results from a population-based survival analysis. Int $\mathrm{J}$ Cancer 108: 761-765, 2004.

20. Han W, Kim SW, Park IA, et al. Young age: an independent risk factor for disease-free survival in women with operabl breast cancer. BMC Cancer 4: 82, 2004.

21. Ozmen V, Ozmen T, Dogru V. Breast cancer in Turkey; An analysis of 20.000 patients with breast cancer. Eur J Breast Health 15: 141-146, 2019.

22. Mougalian SS, Soulos PR, Killelea BK, et al. Use of neoadjuvant chemotherapy for patients with stage I to III breast cancer in the United States. Cancer 121: 2544-2552, 2015.

23. Chen $\mathrm{Y}$, Shi XE, Tian JH, et al. Survival benefit of neoadjuvant chemotherapy for resectable breast cancer. A meta-analysis. Medicine 97: e10634, 2018.

24. Early Breast Cancer Trialists' Collaborative Group (EBCTCG). Long-term outcomes for neoadjuvant versus adjuvant chemotherapy in early breast cancer: meta-analysis of individual patient data from ten randomised trials. Lancet Oncol 19: $27-$

39, 2018.

\section{Coresspondence:}

\section{Dr. Berrin Benli YAVUZ}

Necmettin Erbakan Universitesi, Meram Tip Fakultesi

Radyasyon Onkolojisi Anabilim Dali

Meram, KONYA / TURKEY

Tel: (+90-505) 5150093

e-mail: berrinyavuz77@gmail.com

\section{ORCIDs:}

Berrin Benli Yavuz 0000-0001-7816-4687

Meryem Aktan 0000-0002-0447-7784

Gul Kanyilmaz 0000-0003-2333-353X 